https://doi.org/10.47660/CBR.2020.17197

\title{
OSTEOPOROSIS AND CELIAC DISEASE: A CASE REPORT
}

\begin{abstract}
Lia Poti Gomes Cordeiro',* , João Alexandre Guerra Moita1', Beatrice Araújo Duarte', Lysiane Maria Adeodato Ramos Fontenelle², Carlos Ewerton Maia Rodrigues ${ }^{2}$, Andrea Benevides Leite ${ }^{2}$, Kirla Wagner Poti Gomes ${ }^{2}$
\end{abstract}

1.Centro Universitário Christus, Fortaleza (CE), Brazil. 2.Universidade de Fortaleza, Fortaleza (CE), Brazil.

*Corresponding author: liacoordeiro@gmail.com

\section{BACKGROUND}

Celiac disease (CD) is an autoimmune disorder, triggered by gluten, which causes malabsorption of nutrients and may be a contributing factor to osteoporosis. Often it does not present classically with diarrhea and gastrointestinal symptoms, which makes the early diagnosis of the pathology more difficult. The authors describe the case of a patient with osteoporosis, who was later diagnosed with $\mathrm{CD}$.

\section{CASE REPORT}

A 60-year-old woman presented for rheumatology evaluation due to a 10-year history of osteoporosis. She had a previous lactose intolerance and hyperthyroidism, and had been investigated for lupus due to reagent antinuclear antibody (ANA) test 10 years before. She had menopause at 51 years old, with no hormone replacement. She reported a loss of $4 \mathrm{~kg}$ in the prior 6 months, with no other complaints. On physical examination, only sarcopenia was noted. She had been using zoledronic acid, calcium and vitamin D 7,000 IU/week for 2 years. Laboratory tests showed ANA test 1:160 nuclear dense fine speckled, vitamin D $28 \mathrm{ng} / \mathrm{mL}$, folate $3.5 \mathrm{ng} / \mathrm{mL}$ and vitamin B12 $250 \mathrm{pg} / \mathrm{mL}$; blood count, ESR, CRP, ferritin, serum iron, calcium, phosphorus, alkaline phosphatase, PTH, protein electrophoresis, VDRL, and TSH were normal. Dual-energy X-ray absorptiometry confirmed osteoporosis (T spine score value -4; T femoral neck -2.6). Investigations for malabsorption were carried out and anti-gliadin IgA and anti-transglutaminase IgA antibodies were found to be elevated. Digestive endoscopy was performed, which showed moderate edematous duodenitis; the diagnosis of $C D$ was confirmed by biopsy revealing flat mucosa. She was treated with teriparatide, calcium, vitamin D, folic acid and vitamin B complex; and was instructed to remove gluten from the diet and take regular exercise.

\section{CONCLUSION}

This case shows the importance of considering celiac disease as a cause or aggravating factor for osteoporosis, even in patients without gastrointestinal manifestations and with normal levels of vitamin D and PTH. Recognizing this pathology is necessary to avoid complications of the disease, with nutritional deficiency and osteoporosis. 